\title{
Risk Factors for Asthma in a Helminth Endemic Area in Bahia, Brazil
}

\author{
Luciana S. Cardoso, ${ }^{1,2}$ Daniela M. Costa, ${ }^{1}$ Maria Cecília F. Almeida, ${ }^{1}$ Robson P. Souza, ${ }^{1}$ \\ Edgar M. Carvalho, ${ }^{1,3,4}$ Maria Ilma Araujo, ${ }^{1,3,4}$ and Ricardo R. Oliveira ${ }^{1,5}$ \\ ${ }^{1}$ Serviço de Imunologia, Hospital Universitário Professor Edgard Santos, Universidade Federal da Bahia, \\ 40110-160 Salvador, BA, Brazil \\ ${ }^{2}$ Departamento de Ciências da Vida, Universidade do Estado da Bahia, 41.150-000 Salvador, BA, Brazil \\ ${ }^{3}$ Instituto Nacional de Ciência e Tecnologia de Doenças Tropicais (INCT-DT/CNPq), 40110-160 Salvador, BA, Brazil \\ ${ }^{4}$ Escola Bahiana de Medicina e Saúde Pública, 40290-000 Salvador, BA, Brazil \\ ${ }^{5}$ Faculdade de Farmácia, Universidade Federal da Bahia, 40.170-115 Salvador, BA, Brazil
}

Correspondence should be addressed to Ricardo R. Oliveira, ricardoriccio@gmail.com

Received 14 April 2012; Revised 16 June 2012; Accepted 5 July 2012

Academic Editor: William Harnett

Copyright (C) 2012 Luciana S. Cardoso et al. This is an open access article distributed under the Creative Commons Attribution License, which permits unrestricted use, distribution, and reproduction in any medium, provided the original work is properly cited.

Protective factors associated with atopy or asthma in rural areas include socioeconomic level, overcrowding, and helminth infection. However, little epidemiological information was originated from schistosomiasis areas. This study aimed to investigate factors associated with asthma in a schistosomiasis endemic area. A questionnaire was used to obtain information on demographics, socioeconomic, and environmental features. The ISAAC questionnaire was used to identify individuals with asthma. Parasitological exam was done in all participants and skin prick test to aeroallergens in all asthmatics. Prevalence of Schistosoma mansoni infection was $57.4 \%$ and Ascaris lumbricoides, $30.8 \%$. Asthma was found in $13.1 \%$ of the population, and $35.1 \%$ of them had a positive SPT. Active and passive smoking was positively associated with asthma, whereas A. lumbricoides was negatively associated. In a schistosomiasis hyperendemic region, current infection with A. lumbricoides is protective against asthma. However, we cannot rule out the involvement of $S$. mansoni infection in this process.

\section{Introduction}

Asthma is the most outstanding allergic disease especially in developed countries [1, 2]. However, in developing countries the frequency of asthma is much lower, particularly in rural areas, where the prevalence of parasite infection is high [3-5]. Factors associated with protection against atopy in rural populations include reduced socioeconomic level, poor living conditions, and increased overcrowding [6]. Moreover, several studies have shown a protective role of different parasites in the development of asthma or atopy [6-10].

Studies conducted in Venezuela have shown that geohelminth infections is associated with high levels of total $\operatorname{IgE}$ and low levels of allergen-specific IgE, as well as suppression of allergic reactivity, which is reversible by anthelmintic treatment $[11,12]$. Other authors have also shown low prevalence of positive skin prick test (SPT) for aeroallergens in endemic areas for geohelminths $[7,13]$ or Schistosoma spp. $[9,14]$. We have previously reported that asthmatic individuals living in regions with high frequency of $S$. mansoni infection have a mild course of asthma [15], and that treatment for schistosomiasis leads to a worsening of the clinical manifestations of asthma [3]. It is noteworthy that in these previous studies conducted with $S$. mansoni infected patients, symptoms of asthma or frequency of positive SPT was compared to individuals from outside schistosomiasis endemic areas.

The immunological mechanisms involved with protection against the development of asthma are not well known, although it has been proposed the participation of regulatory molecules [3, 14, 16-20]. Moreover, risk or protective factors associated with asthma in helminth endemic areas remain 
still not well established. Despite a fair amount of epidemiological information is available on risk factors for asthma in developed countries, scant attention has been given to risk factors associated with asthma in rural communities from developing countries, especially in regions endemic for schistosomiasis.

We conducted a cross-sectional study in a rural area endemic for helminths, including S. mansoni, to evaluate possible risk and protective factors associated with the presence of asthma. In addition to demographic, socioeconomic and environmental factors, we also evaluated the influence of current S. mansoni, A lumbricoides, Trichuris trichiura, and hookworm infections with the presence of asthma in individuals living in the region.

\section{Material and Methods}

2.1. Subjects and Endemic Area. This study was carried out at Água Preta, a rural community in the district of Gandu, south of Bahia Brazil, $300 \mathrm{~km}$ distant from Salvador, the capital of the state. This village is composed of about 500 inhabitants, who live in poor sanitary conditions. Using a standard questionnaire, age, sex, income, housing conditions, and exposure to pollutants were registered. Additionally, information on water contact was obtained individually or from guardians in case of children under 10 years old. The International Study of Asthma and Allergies in Childhood (ISAAC) questionnaire, which assess the personal history of bronchial asthma and rhinitis in the past 12 months, as well as family history of atopy and concomitant pathologies, was applied to each individual aging from 6 to 50 years old. In order to exclude individuals with chronic obstructive pulmonary disease (COPD), a clinical evaluation, chest X-ray and spirometry were performed in all asthmatic individuals. None of the asthmatic individuals included in this study had COPD. Subjects who presented personal history of asthma in the past 12 months according to ISAAC questionnaire underwent a skin prick test (SPT) for aeroallergens.

Interviews were carried out initially at the health post of the region, located at the center of Água Preta. The recruitment strategy consisted in household visits to invite subjects to the health post. During the last days of the recruitment, when the number of subjects who spontaneously went to the health post were lower, new household visits were done in order to invite and perform a home-based interview for each individual who agreed in participate in this study.

The ethical Committee of the Maternity Climério de Oliveira, Federal University of Bahia, approved the present study and informed consent was obtained from individual or their legal guardians. All participants who tested positive for parasites were treated at the end of the study. Those who had complaints of allergy were properly instructed regarding indoor environmental control and drug treatment.

2.2. Assessment of Water Contact. The water contact questionnaire was developed based on previous observations carried out on site about habits of the population regarding the use of local rivers. Each participant was asked to provide the frequency and duration of the exposure to the main seven activities (farming, bathing, washing clothes, washing hair, washing dishes, fishing, and playing). Subjects were classified across all activities into four categories to reflect the level of exposure: no exposure, low exposure ( $<1 \mathrm{~h} /$ week), medium exposure (1-3 h/week), or high exposure (1-3 h/day) [21].

2.3. Fecal Examinations for Parasites. All participants received a plastic container for fecal samples at three time points 2-30 days apart. Participants were instructed to deposit stool sample and return the container immediately to the collection point, where samples were stored at $4^{\circ} \mathrm{C}$. Each stool sample had a slide prepared and tested by using the Kato-Katz method to estimate the number of S. mansoni, A. lumbricoides and T. trichiura eggs per gram of feces (EPG) [22]. Additionally, the Hoffmann-Pons-Janer method was used to qualitatively investigate the presence of Hookworm infection [23].

2.4. Skin Prick Test. SPTs were performed on all individuals identified as asthmatic according to the ISAAC criteria. Glycerinated allergens tested included Dermatophagoides pteronyssinus, D. farinae, Blomia tropicalis, Periplaneta Americana, and Blatella germanica (IPI-ASAC). We also used histamine $(1: 1000)$ and saline as positive and negative controls, respectively. SPT results were obtained 20 minutes after application, and a positive skin reaction was defined as a wheal with a mean diameter greater than $3 \mathrm{~mm}$.

2.5. Statistical Analyses. The variable age are expressed as arithmetic mean and standard deviation (SD), whereas parasite load are expressed as geometric mean and 95\% of confidence interval. Helminth infections were classified into low, moderate, or high intensity of infection according to World Health Organization (WHO) criteria [24]. Parasite load ranging from 1 to $99 \mathrm{epg}$ for S. mansoni is classified as low, 100 to 300 epg moderate, and equal or greater than 400 epg high intensity of infection. For A. lumbricoides low intensity of infection is defined as 1 to $4,999 \mathrm{epg}$, moderate 5,000 to $49,999 \mathrm{epg}$, and high intensity of infection is equal or greater than 50,000 epg. Light T. trichiura infection is considered as parasite load ranging from 1 to 999 epg, moderate 1,000 to 9,999 , and high intensity of infection equal or greater than 10,000 epg. The intensity of hookworm infection was not assessed since the Kato-Katz technique is not adequate for quantifying hookworm eggs.

Statistical analyses were performed by using the STATA statistical package version 11.2 (Stata Corp., College Station, TX). A logistic regression model was used to test association between asthma and covariates in a univariate and multivariate way. The alpha level for statistical significance was established as 0.05 for all analyses and odds ratio (OR) was obtained at a $95 \%$ of confidence interval (CI). In order to reduce the chance of type I error a $P$ value adjustment was performed by the Bonferroni correction in all significant associations. Multivariate analyses were adjusted for age, gender, number of stool samples evaluated, and/or smoking status. Adjustment for age and gender was performed because these could be possible confounding 
factors, since depending on gender or age lifestyle could be significantly different. The adjustment for number of stool samples analyzed was done because $82.8 \%$ of subjects who had three samples evaluated were asthmatics, and it is well known that the Kato-Katz sensitivity is influenced by the number of samples evaluated, reaching a sensitivity of $91.7 \%$ for the diagnosis of schistosomiasis when four samples are analyzed [25]. For investigating the influence of helminth infections on asthma, the smoking status was taken into account since this was positively associated with asthma in this region, which could be responsible for the underestimation of the real effect that helminth infections have on asthma.

\section{Results}

3.1. Baseline Characteristics of the Studied Subjects. A total of 427 individuals were included in this study, being 53.2\% male. The mean age was $26.9 \pm 19.0$ years, ranging from 1 to 92 years. Approximately $95 \%$ of participants reported an average income less than the Brazilian national minimum wage $(\leq \$ 250.00)$ per month. Regarding the presence of pollutants $94.4 \%$ of the population uses wood burning stove and $14.5 \%$ were active smokers.

The frequency of intestinal parasites was investigated in 406 (95.1\%) individuals, 214 (52.7\%) of whom had only one sample analyzed, whereas $160(39.4 \%)$ and $32(7.9 \%)$ subjects had two and three samples evaluated, respectively. The helminth most frequently identified in the region was $S$. mansoni, affecting $57.4 \%$ of the population, followed by $T$. trichiura (36.9\%), hookworm (31.8\%), and A. lumbricoides $(30.8 \%)$. Intensity of infection was assessed for S. mansoni (89 [75-105] epg), A. lumbricoides (1,946 [1,278-2,963] epg), and T. trichiura (120 [97-149] epg).

History of exposure to contaminated water was reported by $332(77.8 \%)$ subjects, $34.3 \%$ of whom had a low level of exposure, $18.7 \%$ medium, and $47.0 \%$ were highly exposed. The group highly exposed was composed mainly of female $(67.9 \%)$, whereas the group with low level of exposure was represented mostly by men $(68.4 \%)$. Despite the evident influence of gender on the intensity of exposure, age is a factor that apparently did not affect the level of exposure. The group classified as highly exposed had a mean age of $27 \pm 17$ years, while the groups of medium and low level of exposure had a mean age of $26 \pm 18$ and $27 \pm 20(P>0.05)$, respectively. As expected, the group that reported higher level of exposure also showed higher frequency of schistosomiasis, when compared to the nonexposed group (Table 1).

The prevalence of asthma and rhinitis was evaluated by the ISAAC questionnaire in 335 individuals, representing all subjects who were 6 to 50 years old. The prevalence of selfreported wheezing in the past 12 months (here designated as "asthma") was $13.1 \%$. Skin prick test for aeroallergens was performed on 37 out of $44(84.1 \%)$ asthmatic individuals. False positive result, defined as presence of skin reaction in the negative control saline, was found in only one subject $(2.3 \%)$. On the other hand, false negative result, defined as no skin reaction detected, even to histamine, was found in four (9.1\%) asthmatic individuals. Among those 32 individuals who had a validated skin prick test result, 13 (35.1\%) was positive for at least one allergen, whereas 19 (64.9\%) of them had a negative SPT result.

3.2. Risk Factors Associated with Asthma. Univariate and multivariate analysis for the association between asthma and possible risk or protector factors are presented in Table 2 . Evaluated variables included gender, age, income, passive or active smoking, family history of allergy, housing quality, number of people per household, use of wood burning stoves, and level of exposure to fresh water. Multivariate analyses were adjusted for age and gender.

The frequency of asthmatic individuals living in the endemic area who are active smokers was higher (26.2\%) than the prevalence of active smokers among those nonasthmatic subjects $(11.8 \%)$. In order to obtain the power of this difference, we performed a sample size calculation based on these data and found that 42 individuals in the "Asthmatic" group and 272 in the "nonasthmatic" group have $76.1 \%$ power to detect a difference with a significance alpha level of 0.05 .

Only active or passive smoking was positively associated with the presence of personal history of asthma in the past 12 months, even after adjustment for confounding factors (Table 2). After controlling for age and gender the prevalence of asthma was 5.45 (CI 95\%: 2.09-14.24; $P=0.001$ ) and 3.67 (CI 95\%: 1.83-7.34; $P<0.001)$ times higher among active and passive smokers, respectively, than nonsmokers.

\subsection{Association between Asthma and Helminth Infections.} The association between helminth infection or intensity of infection and asthma is shown in Table 3. Univariate analysis indicates that hookworm infection $(\mathrm{OR}=2.54$ [1.32-4.91]; $P=0.005)$, moderate intensity of $A$. lumbricoides infection $(\mathrm{OR}=4.01[1.74-9.28] ; P=0.001)$, T. trichiura $(\mathrm{OR}=2.18$ [1.13-4.20]; $P=0.020$ ), and S. mansoni (OR $=2.89$ [1.296.48]; $P=0.010$ ) infection were positively associated with asthma. However when adjusting for the confounding factors age, gender, number of stool samples evaluated, and smoking status, no significant association was observed between hookworm $(\mathrm{OR}=1.41[0.54-3.72] ; P=0.486)$, T. trichiura $(\mathrm{OR}=0.95[0.36-2.54] ; P=0.918)$, S. mansoni $(\mathrm{OR}=1.44$ [0.48-4.29]; $P=0.517)$, or any helminth infection (OR $=0.94[0.19-4.56] ; P=0.935)$ and asthma. Moreover, no significant association was found between number of helminth infections and asthma (data not shown).

Multivariate analysis showed that $A$. lumbricoides infection is negatively associated with asthma $(\mathrm{OR}=0.26[0.07-$ 0.94]; $P=0.041)$. When the Bonferroni correction was performed this association became nonsignificant $(P=0.984)$. Nevertheless, we found a significant association between A. lumbricoides infection and asthma when controlling for other helminth infections (data not shown). The association between asthma and parasite load was also assessed and we found that low $(\mathrm{OR}=0.27[0.07-0.94] P=0.071)$ or moderate $(\mathrm{OR}=0.29[0.05-1.83] ; P=0.187)$ intensity of A. lumbricoides infection was not associated with asthma. Since only three individuals, all nonasthmatic, showed high intensity of A. lumbricoides infection in the region, we were 
TABLE 1: Frequency of S. mansoni infection according to the level of exposure.

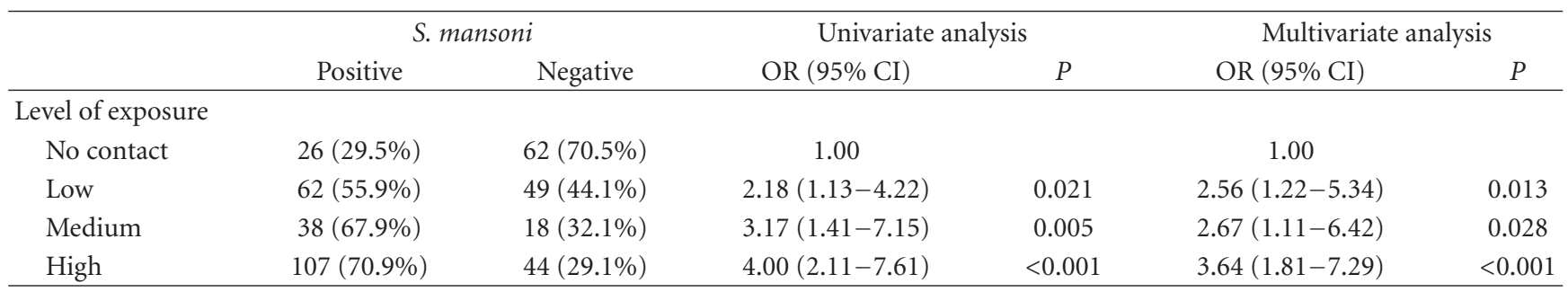

Note: Multivariate analysis adjusted by age, gender, and number of stool samples analyzed.

TABLE 2: Univariate and multivariate analysis for the association between asthma and possible risk or protector factors.

\begin{tabular}{|c|c|c|c|c|c|c|}
\hline \multirow{3}{*}{ Gender } & \multirow{2}{*}{$\begin{array}{c}\text { Nonasthmatic } \\
\quad(n=291)\end{array}$} & \multirow{2}{*}{$\begin{array}{c}\text { Asthmatic } \\
(n=44)\end{array}$} & \multicolumn{2}{|c|}{ Univariate analysis } & \multicolumn{2}{|c|}{ Multivariate analysis } \\
\hline & & & OR $(95 \% \mathrm{CI})$ & $P$ & OR $(95 \% \mathrm{CI})$ & $P$ \\
\hline & & & & & & \\
\hline Female & $141(85.4 \%)$ & $24(14.6 \%)$ & 1.00 & & & \\
\hline Male & $150(88.2 \%)$ & $20(11.8 \%)$ & $0.78(0.41-1.48)$ & 0.452 & & \\
\hline Age (years) & & & & & & \\
\hline $6-10$ & $44(83.0 \%)$ & $9(17.0 \%)$ & 1.00 & & & \\
\hline $11-15$ & $48(81.4 \%)$ & $11(18.6 \%)$ & $1.12(0.42-2.96)$ & 0.819 & & \\
\hline $16-20$ & $41(91.1 \%)$ & $4(8.9 \%)$ & $0.48(0.14-1.67)$ & 0.247 & & \\
\hline $21-30$ & $82(88.2 \%)$ & $11(11.8 \%)$ & $0.66(0.25-1.70)$ & 0.386 & & \\
\hline $31-40$ & $43(89.6 \%)$ & $5(10.4 \%)$ & $0.57(0.18-1.83)$ & 0.345 & & \\
\hline $41-50$ & $33(89.2 \%)$ & $4(10.8 \%)$ & $0.59(0.17-2.09)$ & 0.416 & & \\
\hline Income $(\$)$ & & & & & & \\
\hline$<250$ & $271(86.9 \%)$ & $41(13.1 \%)$ & 1.00 & & 1.00 & \\
\hline $250-500$ & $19(86.4 \%)$ & $3(13.6 \%)$ & $1.04(0.30-3.68)$ & 0.947 & $1.14(0.31-4.17)$ & 0.847 \\
\hline$>500$ & 0 & 0 & - & - & - & - \\
\hline Active smoker & & & & & & \\
\hline No & $240(88.6 \%)$ & $31(11.4 \%)$ & 1.00 & & 1.00 & \\
\hline Yes & $32(74.4 \%)$ & $11(25.6 \%)$ & $2.66(1.22-5.81)$ & 0.014 & $5.45(2.09-14.24)$ & 0.001 \\
\hline Passive smoker & & & & & & \\
\hline No & $190(92.7 \%)$ & $15(7.3 \%)$ & 1.00 & & 1.00 & \\
\hline Yes & $97(78.2 \%)$ & $27(21.2 \%)$ & $3.53(1.79-6.94)$ & $<0.001$ & $3.67(1.83-7.34)$ & $<0.001$ \\
\hline Family history o & & & & & & \\
\hline No & $180(87.0 \%)$ & $27(13.0 \%)$ & 1.00 & & 1.00 & \\
\hline Yes & $65(84.4 \%)$ & $12(15.6 \%)$ & $1.23(0.59-2.57)$ & 0.581 & $1.29(0.60-2.77)$ & 0.508 \\
\hline Housing quality & & & & & & \\
\hline Brick house & $103(92.0 \%)$ & $9(8.0 \%)$ & 1.00 & & 1.00 & \\
\hline Adobe house & $107(84.3 \%)$ & $20(15.7 \%)$ & $2.14(0.93-4.92)$ & 0.073 & $2.03(0.88-4.72)$ & 0.099 \\
\hline Mud house & $48(87.3 \%)$ & $7(12.7 \%)$ & $1.67(0.59-4.77)$ & 0.337 & $1.77(0.61-5.08)$ & 0.290 \\
\hline Number of inhal & & & & & & \\
\hline $1-3$ & $95(87.2 \%)$ & $14(12.8 \%)$ & 1.00 & & 1.00 & \\
\hline $4-6$ & $148(88.1 \%)$ & $20(11.9 \%)$ & $0.92(0.44-1.90)$ & 0.816 & $0.76(0.35-1.64)$ & 0.485 \\
\hline$>6$ & $43(81.1 \%)$ & $10(18.9 \%)$ & $1.58(0.65-3.83)$ & 0.314 & $1.40(0.56-3.52)$ & 0.475 \\
\hline Wood burning s & & & & & & \\
\hline No & $19(95.0 \%)$ & $1(5.0 \%)$ & 1.00 & & 1.00 & \\
\hline Yes & $271(86.3 \%)$ & $43(13.7 \%)$ & $3.01(0.39-23.1)$ & 0.288 & $2.83(0.37-21.88)$ & 0.319 \\
\hline Exposure to fres & & & & & & \\
\hline No contact & $57(87.7 \%)$ & $8(12.3 \%)$ & 1.00 & & 1.00 & \\
\hline Low & $86(93.5 \%)$ & $6(6.5 \%)$ & $0.50(0.16-1.51)$ & 0.217 & $0.48(0.15-1.50)$ & 0.207 \\
\hline Medium & $41(85.4 \%)$ & $7(14.6 \%)$ & $1.22(0.41-3.62)$ & 0.725 & $1.26(0.42-3.81)$ & 0.684 \\
\hline High & $107(82.3 \%)$ & $23(17.7 \%)$ & $1.53(0.64-3.64)$ & 0.335 & $1.60(0.66-3.87)$ & 0.293 \\
\hline
\end{tabular}

Note: Multivariate analysis adjusted by age and gender. 
TABLE 3: Univariate and multivariate analysis of the association between asthma and helminth infections.

\begin{tabular}{|c|c|c|c|c|c|c|}
\hline & \multirow{2}{*}{$\begin{array}{c}\text { Nonasthmatic } \\
(n=291) \\
33 \%\end{array}$} & \multirow{2}{*}{$\begin{array}{c}\text { Asthmatic } \\
(n=42) \\
45 \%\end{array}$} & \multicolumn{2}{|c|}{ Univariate analysis } & \multicolumn{2}{|c|}{ Multivariate analysis } \\
\hline & & & OR $(95 \% \mathrm{CI})$ & $P$ & OR $(95 \% \mathrm{CI})$ & $P$ \\
\hline \multicolumn{7}{|l|}{ Hookworm } \\
\hline Negative & $189(90.9 \%)$ & $19(9.1 \%)$ & 1.00 & & 1.00 & \\
\hline Positive & $90(79.6 \%)$ & $23(20.4 \%)$ & $2.54(1.32-4.91)$ & 0.005 & $1.41(0.54-3.72)$ & 0.486 \\
\hline \multicolumn{7}{|l|}{ A. lumbricoides } \\
\hline Negative & $187(89.0 \%)$ & $23(11.0 \%)$ & 1.00 & & 1.00 & \\
\hline Positive & $92(82.9 \%)$ & $19(17.1 \%)$ & $1.68(0.87-3.24)$ & 0.122 & $0.26(0.07-0.94)$ & 0.041 \\
\hline \multicolumn{7}{|l|}{ Intensity (epg) } \\
\hline 0 & $193(89.4 \%)$ & $23(10.6 \%)$ & 1.00 & & 1.00 & \\
\hline $1-4999$ & $60(88.2 \%)$ & $8(11.8 \%)$ & $1.12(0.48-2.63)$ & 0.797 & $0.27(0.07-1.12)$ & 0.071 \\
\hline 5000-49999 & $23(67.6 \%)$ & $11(32.4 \%)$ & $4.01(1.74-9.28)$ & 0.001 & $0.29(0.05-1.83)$ & 0.187 \\
\hline$\geq 50000$ & $3(100 \%)$ & 0 & - & - & - & - \\
\hline \multicolumn{7}{|l|}{ T. trichiura } \\
\hline Negative & $173(90.6 \%)$ & $18(9.4 \%)$ & 1.00 & & 1.00 & \\
\hline Positive & $106(81.5 \%)$ & $24(18.5 \%)$ & $2.18(1.13-4.20)$ & 0.020 & $0.95(0.36-2.54)$ & 0.918 \\
\hline \multicolumn{7}{|l|}{ Intensity (epg) } \\
\hline 0 & $175(90.7 \%)$ & $18(9.3 \%)$ & 1.00 & & 1.00 & \\
\hline $1-999$ & $96(80.7 \%)$ & $23(19.3 \%)$ & $2.33(1.20-4.53)$ & 0.013 & $1.02(0.38-2.74)$ & 0.968 \\
\hline 1000-9999 & $7(87.5 \%)$ & $1(12.5 \%)$ & $1.39(1.61-11.9)$ & 0.765 & $0.30(0.01-8.03)$ & 0.474 \\
\hline$\geq 10000$ & $1(100 \%)$ & 0 & - & - & - & - \\
\hline \multicolumn{7}{|l|}{ S. mansoni } \\
\hline Negative & $113(93.4 \%)$ & $8(6.6 \%)$ & 1.00 & & 1.00 & \\
\hline Positive & $166(83.0 \%)$ & $34(17.0 \%)$ & $2.89(1.29-6.48)$ & 0.010 & $1.44(0.48-4.29)$ & 0.517 \\
\hline \multicolumn{7}{|l|}{ Intensity (epg) } \\
\hline 0 & $114(93.4 \%)$ & $8(6.6 \%)$ & 1.00 & & 1.00 & \\
\hline $1-99$ & $104(86.7 \%)$ & $16(13.3 \%)$ & $2.19(0.90-5.33)$ & 0.084 & $1.48(0.45-4.84)$ & 0.520 \\
\hline $100-399$ & $34(73.9 \%)$ & $12(26.1 \%)$ & $5.03(1.90-13.3)$ & 0.001 & $3.21(0.76-13.63)$ & 0.114 \\
\hline$\geq 400$ & $27(81.8 \%)$ & $6(18.2 \%)$ & $3.17(1.01-9.89)$ & 0.047 & $0.69(0.10-4.82)$ & 0.706 \\
\hline \multicolumn{7}{|l|}{ Any helminth } \\
\hline Negative & $49(94.2 \%)$ & $3(5.8 \%)$ & 1.00 & & 1.00 & \\
\hline Positive & $230(85.5 \%)$ & $39(14.5 \%)$ & $2.77(0.82-9.33)$ & 0.100 & $0.94(0.19-4.56)$ & 0.935 \\
\hline
\end{tabular}

Note: Multivariate analysis adjusted by age, gender, number of stool samples analyzed, and smoking status.

not able to test the association between high ascariasis parasite load and asthma.

\section{Discussion}

This study assessed possible risk or protective factors associated with presence of asthma in a population living in a rural poor area of Bahia, Brazil. Our results showed that the main risk factor associated with presence of asthma is smoking. We found no association between socioeconomic variables and the development of asthma. Additionally, we demonstrated that current $A$. lumbricoides infection is associated with protection against asthma when adjusting for confounding factors.

The cross-sectional design, the use of questionnaires to access information on the presence of asthma, and risk factors were the major limitations of this study. The study design did not allow us to make causal inferences, and confounding factors may not be equally distributed between groups. However, we performed statistical analysis taking into account the confounding factors, and reverse causality is not possible for most of the risk factors evaluated. We are also aware that the use of questionnaires makes the study open to information bias since it depends on the interviewed response. Therefore, misclassification of exposure may have occurred in the present study. Also, we call attention that we did not evaluate past helminth infections and that the parasitological technique used here is not sensitive for very low parasite burden.

The differentiation between asthma and COPD was one of our major concerns when planning this study. One way to reduce this possible misdiagnose was the exclusion of 
individuals over 50 years of age. Many authors have reported that COPD rarely presents clinically before the 5th decade of life and that the prevalence of COPD occurs primarily in smokers over 40 years of age $[26,27]$. According to the PLATINO study the prevalence of COPD in individuals aging from 40 to 49 years in a metropolitan area of Brazil is $8.4 \%$ [28]. In our study only $4(9 \%)$ asthmatic individuals aged between 41 and 50 years old. Therefore, 91\% of subjects included in this study were under 41 years old. Moreover, only 1 out of 4 (25\%) asthmatic individual over 40 years of age was active smoker.

Although we did not observe association between socioeconomic variables and asthma, other authors have demonstrated that reduced socioeconomic level and overcrowding are associated with protection against atopy in a rural region [6]. This phenomenon was probably not detected in our study because the overall population was homogeneous in regard to socioeconomic level, being identified only subjects with an average income less than $\$ 500$ per month. Additionally, several other studies have showed an association between smoking and risk of asthma, which is consistent with our data [29-32]. Even though this association is well known, its interpretation must be made cautiously, especially when using a questionnaire-based diagnosis of asthma. Because smokers have been reported to have more severe asthma than nonsmokers, it is expected that the use of selfreported questionnaires, such as the ISAAC questionnaire, may overestimate the risk of asthma among smokers [33]. However, this bias probably did not interfere in our study since it has been shown that individuals from endemic areas for schistosomiasis have mild asthma [15]. In order to evaluate the effect of helminth infections in the presence of asthma statistical analyses were adjusted for smoking status, given that this is an independent determinant factor for asthma.

In the last years researchers showed an increased interest in evaluating the role of helminth infections in the development of allergic diseases. Although several studies show that in endemic areas for helminths, infection with $A$. lumbricoides or other helminths has a negative association with atopy, possibly acting as a protective factor against the development of asthma, none of these studies was conducted in endemic area for schistosomiasis [6-8, 12, 34]. Studies carried out with individuals from endemic areas infected with S. mansoni have showed low reactivity to the skin prick test (SPT) to aeroallergens and fewer symptoms of asthma compared to asthmatics without infection [10, 15], and an inverse correlation between SPT and presence of infection [9]. It is worth noting that none of these previous studies evaluated the association between SPT or symptoms of asthma and current $S$. mansoni infection within asthmatic subjects living in the same region. The prevalence of positive SPT to aeroallergens in asthmatic individuals included in this study was $35.1 \%$, which is far below what is found in urban regions from Brazil [10, 35, 36]. Nevertheless, we found no significant difference in the frequency of positive SPT among asthmatics currently infected or not by any helminth (data not shown). Despite the frequency of positive skin test has been low, asthmatic subjects from these regions probably have atopic asthma, demonstrated by the presence of aeroallergen-specific IgE [10].

The presence of asthma was not associated with current infection by $S$. mansoni in the studied population. However, the prevalence of asthma in the site was only $13.1 \%$, which is much lower than the frequency observed in adolescents of 13 and 14 years old from urban areas of Salvador $(24.6 \%)$, Feira de Santana $(21.5 \%)$ or Vitoria da Conquista $(30.5 \%)$, Bahia, when using the same criteria for defining asthma [5]. The prevalence of asthma was representative of the entire study population, including individuals from 6 to 50 years old. However, when analyzing only the age of 13 and 14 years of age, the prevalence was $15 \%$, which is very close to the frequency observed in the general population in the endemic area and still far from that observed in other centers evaluated in Bahia, Brazil [5]. This is in agreement with other study conducted in different regions of Brazil, which showed lower prevalence of asthma and rhinitis in rural than in urban areas [4].

Thus, the presence of a protective factor for the development of asthma in an endemic community for helminths including schistosomiasis is evident, which could not be explained only by the presence of current S. mansoni infection. The fact that these individuals inhabit a region of high endemicity, constantly exposed to S. mansoni reinfection, might be responsible for the low prevalence of asthma observed on site, with no significant difference in the frequency of asthma among currently infected or not. On the other hand, we found in this schistosomiasis endemic area that individuals currently infected with A. lumbricoides have a lower prevalence of asthma when compared to noninfected subjects, which was not observed for the presence of $T$. trichiura, hookworm, any helminth infection, or even for different intensity of infection. Although this association has been lost when performing the Bonferroni correction, it is well established that reducing type I error by $P$ value adjustments increases the probability of type II error for those association that are not null. The formal premise for such adjustments is the much wider hypothesis that there is no association between any variables under observation, and that only random processes govern the variability of all observations. This "universal" null hypothesis presumes that all observed associations reflect only random variation that could be obtained particularly in empiric situations $[37,38]$. Once this study evaluated associations that are not empiric, but instead evidence-based, such as the association between A. lumbricoides infection and asthma, we are confident that this association was not due to random variation, and therefore it would be better to take the risk of making type I error and call attention to a possible beneficial association for asthma.

Studies conducted in urban areas in Brazil have showed controversial results for the association between A. lumbricoides infection and atopy $[8,39]$. Although no association between SPT response and A. lumbricoides infection was found in asthmatic individuals [39], a recent study showed that the presence of $A$. lumbricoides infection is able to reduce the prevalence of positive SPT response but do not affect the prevalence of asthma [8]. Overall, helminth infection is 
associated with reduced risk of asthma or atopy in regions with high frequency of parasite infections $[7,11,13]$, whereas in areas with low endemicity, A. lumbricoides infection is associated with increased risk of atopy and asthma [40, 41]. Although A. lumbricoides is a recognized cause of tropical pulmonary eosinophilia, chronic infection induces a regulatory immune response which includes interleukin-10and transforming growth factor- $\beta$-secreting cells [42, 43]. In addition to the suppression of allergic inflammation, activation of the regulatory immune response induced by helminths is responsible for preventing the elimination of parasites and protects the host from damage that could be caused by excessive inflammatory response [44]. Our findings support the hypothesis that exposure to A. lumbricoides induces a generalized suppression on the immune response, which seems to be able to reduce the risk of asthma without additive effect by helminth coinfection. This effect was not observed for other helminth infections probably because $A$. lumbricoides was the less frequently helminth found in the region, which results in less exposure and lower rates of reinfection, allowing thereby the detection of differences in asthma prevalence between individuals currently infected or not.

In conclusion, our findings indicate that smoking is the major risk factors associated with asthma in a rural population endemic for schistosomiasis. However, in rural endemic areas for helminth infections the prevalence of asthma is much lower than what is observed in urban regions, and A. lumbricoides infection is negatively associated with the presence of asthma within the population. Although we were unable to show a protective association between current S. mansoni infection and asthma, we cannot rule out the possible participation of $S$. mansoni in modulating the immune response of allergic diseases, probably because this is a hyperendemic region where individuals are constantly reinfected. This study contribute to a better understanding of the role of helminth infections in allergies.

\section{Conflict of Interests}

The authors have no conflict of interests concerning the work reported in this paper.

\section{Acknowledgments}

The authors thank Lêda Maria Alcântara for the collaboration in reading parasitological slides; all individuals from the region of Água Preta-Gandu, Bahia, Brazil, for participating in the study; the Health Agent Irene de Jesus for collaborating in the field work.

\section{References}

[1] R. Beasley, U. Keil, E. Von Mutius, and N. Pearce, "Worldwide variation in prevalence of symptoms of asthma, allergic rhinoconjunctivitis, and atopic eczema: ISAAC," The Lancet, vol. 351, no. 9111, pp. 1225-1232, 1998.

[2] M. I. Asher, "Worldwide variations in the prevalence of asthma symptoms: the International Study of Asthma and Allergies in Childhood (ISAAC)," European Respiratory Journal, vol. 12, no. 2, pp. 315-335, 1998.

[3] M. I. A. S. Araujo, B. Hoppe, M. Medeiros et al., "Impaired $\mathrm{T}$ helper 2 response to aeroallergen in helminth-infected patiente with asthma," Journal of Infectious Diseases, vol. 190, no. 10, pp. 1797-1803, 2004.

[4] D. Solé, V. E. Cassol, A. R. Silva et al., "Prevalence of symptoms of asthma, rhinitis, and atopic eczema among adolescents living in urban and rural areas in different regions of Brazil," Allergologia et Immunopathologia, vol. 35, no. 6, pp. 248-253, 2007.

[5] D. Solé, G. F. Wandalsen, I. C. Camelo-Nunes et al., "Prevalence of symptoms of asthma, rhinitis, and atopic eczema among Brazilian children and adolescents identified by the International Study of Asthma and Allergies in Childhood (ISAAC)_Phase 3," Jornal de Pediatria, vol. 82, no. 5, pp. 341346, 2006.

[6] P. J. Cooper, M. E. Chico, L. C. Rodrigues et al., "Risk factors for atopy among school children in a rural area of Latin America," Clinical and Experimental Allergy, vol. 34, no. 6, pp. 845-852, 2004.

[7] P. J. Cooper, M. E. Chico, L. C. Rodrigues et al., "Reduced risk of atopy among school-age children infected with geohelminth parasites in a rural area of the tropics," Journal of Allergy and Clinical Immunology, vol. 111, no. 5, pp. 995-1000, 2003.

[8] N. M. Alcantara-Neves, R. V. Veiga, V. C. C. Dattoli et al., "The effect of single and multiple infections on atopy and wheezing in children," Journal of Allergy and Clinical Immunology, vol. 129, no. 2, pp. 359.e1-367.e3, 2012.

[9] M. I. Araujo, A. A. Lopes, M. Medeiros et al., "Inverse association between skin response to aeroallergens and Schistosoma mansoni infection," International Archives of Allergy and Immunology, vol. 123, no. 2, pp. 145-148, 2000.

[10] M. Medeiros, M. C. Almeida, J. P. Figueiredo et al., "Low frequency of positive skin tests in asthmatic patients infected with Schistosoma mansoni exposed to high levels of mite allergens," Pediatric Allergy and Immunology, vol. 15, no. 2, pp. 142-147, 2004.

[11] N. R. Lynch, I. Hagel, M. Perez, M. C. Di Prisco, R. Lopez, and N. Alvarez, "Effect of anthelmintic treatment on the allergic reactivity of children in a tropical slum," Journal of Allergy and Clinical Immunology, vol. 92, no. 3, pp. 404-411, 1993.

[12] N. R. Lynch, R. I. Lopez, and M. C. Di Prisco-Fuenmayor, "Allergic reactivity and socio-economic level in a tropical environment," Clinical Allergy, vol. 17, no. 3, pp. 199-207, 1987.

[13] P. J. Cooper, M. E. Chico, M. Bland, G. E. Griffin, and T. B. Nutman, "Allergic symptoms, atopy, and geohelminth infections in a rural area of Ecuador," American Journal of Respiratory and Critical Care Medicine, vol. 168, no. 3, pp. 313317, 2003.

[14] A. H. J. Van den Biggelaar, R. Van Ree, L. C. Rodrigues et al., "Decreased atopy in children infected with Schistosoma haematobium: a role for parasite-induced interleukin-10," The Lancet, vol. 356, no. 9243, pp. 1723-1727, 2000.

[15] M. Medeiros, J. P. Figueiredo, M. C. Almeida et al., "Schistosoma mansoni infection is associated with a reduced course of asthma," Journal of Allergy and Clinical Immunology, vol. 111, no. 5, pp. 947-951, 2003.

[16] L. G. G. Pacífico, F. A. V. Marinho, C. T. Fonseca et al., "Schistosoma mansoni antigens modulate experimental allergic asthma in a murine model: a major role for $\mathrm{CD} 4^{+} \mathrm{CD} 25^{+}$Foxp $^{+} \mathrm{T}$ 
cells independent of interleukin-10," Infection and Immunity, vol. 77, no. 1, pp. 98-107, 2009.

[17] E. Mitre, S. Norwood, and T. B. Nutman, "Saturation of immunoglobulin E (IgE) binding sites by polyclonal IgE does not explain the protective effect of helminth infections against atopy," Infection and Immunity, vol. 73, no. 7, pp. 4106-4111, 2005.

[18] R. R. Oliveira, K. J. Gollob, J. P. Figueiredo et al., "Schistosoma mansoni infection alters co-stimulatory molecule expression and cell activation in asthma," Microbes and Infection, vol. 11, no. 2, pp. 223-229, 2009.

[19] M. Yazdanbakhsh and P. M. Matricardi, "Parasites and the hygiene hypothesis: regulating the immune system?" Clinical Reviews in Allergy and Immunology, vol. 26, no. 1, pp. 15-23, 2004.

[20] L. S. Cardoso, S. C. Oliveira, A. M. Góes et al., "Schistosoma mansoni antigens modulate the allergic response in a murine model of ovalbumin-induced airway inflammation," Clinical and Experimental Immunology, vol. 160, no. 2, pp. 266-274, 2010.

[21] A. J. Dessein, M. Begley, C. Demeure et al., "Human resistance to Schistosoma mansoni is associated with IgG reactivity to a $37-\mathrm{kDa}$ larval surface antigen," The Journal of Immunology, vol. 140, no. 8, pp. 2727-2736, 1988.

[22] N. Katz, P. M. Coelho, and J. Pellegrino, "Evaluation of Kato's quantitative method through the recovery of Schistosoma mansoni eggs added to human feces," Journal of Parasitology, vol. 56, no. 5, pp. 1032-1033, 1970.

[23] W. A. Hoffman, J. A. Pons, and J. L. Janer, "Sedimentation concentration method in Schistosomiasis mansoni," Puerto Rico Journal of Public Health, vol. 9, pp. 283-298, 1934.

[24] WHO, Prevention and Control of Schistosomiasis and SoilTransmitted Helminthiasis, Technical Report Series No. 912, World Health Organization, 2002.

[25] A. Ebrahim, H. El-Morshedy, E. Omer, S. El-Daly, and R. Barakat, "Evaluation of the Kato-Katz thick smear and formol ether sedimentation techniques for quantitative diagnosis of Schistosoma mansoni infection," American Journal of Tropical Medicine and Hygiene, vol. 57, no. 6, pp. 706-708, 1997.

[26] P. Kardos and J. Keenan, "Tackling COPD: a multicomponent disease driven by inflammation," MedGenMed Medscape General Medicine, vol. 8, no. 3, article 54, 2006.

[27] E. D. Bateman, C. Feldman, J. O’Brien et al., "Guideline for the management of chronic obstructive pulmonary disease (COPD): 2004 revision," South African Medical Journal, vol. 94, no. 7, part 2, pp. 559-575, 2004.

[28] A. M. B. Menezes, R. Perez-Padilla, J. R. B. Jardim et al., "Chronic obstructive pulmonary disease in five Latin American cities (the PLATINO study): a prevalence study," The Lancet, vol. 366, no. 9500, pp. 1875-1881, 2005.

[29] K. Torén, T. Gislason, E. Omenaas et al., "A prospective study of asthma incidence and its predictors: the RHINE study," European Respiratory Journal, vol. 24, no. 6, pp. 942-946, 2004.

[30] X. Wang, T. T. Mensinga, J. P. Schouten, B. Rijcken, and S. T. Weiss, "Determinants of maximally attained level of pulmonary function," American Journal of Respiratory and Critical Care Medicine, vol. 169, no. 8, pp. 941-949, 2004.

[31] T. Sigsgaard, C. Hjort, Ø. Omland, M. R. Miller, and O. F. Pedersen, "Respiratory health and allergy among young farmers and non-farming rural males in Denmark: the SUS study," Journal of Agromedicine, vol. 9, no. 2, pp. 223-238, 2004.
[32] Ø. Omland, C. Hjort, O. F. Pedersen, M. R. Miller, and T. Sigsgaard, "New-onset asthma and the effect of environment and occupation among farming and nonfarming rural subjects," Journal of Allergy and Clinical Immunology, vol. 128, no. 4, pp. 761-765, 2011.

[33] C. Svanes, E. Omenaas, G. E. Eide, O. Fluge, and A. Gulsvik, "Hospitalization for lung disease in early childhood and asthma symptoms in young adulthood," Respiratory Medicine, vol. 92, no. 8, pp. 1003-1009, 1998.

[34] N. R. Lynch, I. A. Hagel, M. E. Palenque et al., "Relationship between helminthic infection and IgE response in atopic and nonatopic children in a tropical environment," Journal of Allergy and Clinical Immunology, vol. 101, no. 2, part 1, pp. 217-221, 1998.

[35] A. C. Pastorino, R. D. C. Rimazza, C. Leone, A. P. M. Castro, D. Solé, and C. M. A. Jacob, "Risk factors for asthma in adolescents in a large urban region of Brazil," Journal of Asthma, vol. 43, no. 9, pp. 695-700, 2006.

[36] A. C. Pastorino, F. C. Kuschnir, L. K. P. Arruda et al., "Sensitisation to aeroallergens in Brazilian adolescents living at the periphery of large subtropical urban centres," Allergologia et Immunopathologia, vol. 36, no. 1, pp. 9-16, 2008.

[37] K. J. Rothman, "No adjustments are needed for multiple comparisons," Epidemiology, vol. 1, no. 1, pp. 43-46, 1990.

[38] T. V. Perneger, "What's wrong with Bonferroni adjustments," BMJ, vol. 316, no. 7139, pp. 1236-1238, 1998.

[39] E. Vieira Ponte, F. Lima, M. I. Araújc, R. Riccio Oliveira, L. Santos Cardoso, and A. A. Cruz, "Skin test reactivity and Der p-induced interleukin 10 production in patients with asthma or rhinitis infected with Ascaris," Annals of Allergy, Asthma and Immunology, vol. 96, no. 5, pp. 713-718, 2006.

[40] G. M. Hunninghake, M. E. Soto-Quiros, L. Avila et al., "Sensitization to Ascaris lumbricoides and severity of childhood asthma in Costa Rica," Journal of Allergy and Clinical Immunology, vol. 119, no. 3, pp. 654-661, 2007.

[41] L. J. Palmer, J. C. Celedón, S. T. Weiss, B. Wang, Z. Fang, and X. $\mathrm{Xu}$, "Ascaris lumbricoides infection is associated with increased risk of childhood asthma and atopy in rural China," American Journal of Respiratory and Critical Care Medicine, vol. 165, no. 11, pp. 1489-1493, 2002.

[42] C. A. Figueiredo, M. L. Barreto, L. C. Rodrigues et al., "Chronic intestinal helminth infections are associated with immune hyporesponsiveness and induction of a regulatory network," Infection and Immunity, vol. 78, no. 7, pp. 31603167, 2010.

[43] J. D. Turner, J. A. Jackson, H. Faulkner et al., "Intensity of intestinal infection with multiple worm species is related to regulatory cytokine output and immune hyporesponsiveness," Journal of Infectious Diseases, vol. 197, no. 8, pp. 1204-1212, 2008.

[44] R. M. Maizels and M. Yazdanbakhsh, "Immune regulation by helminth parasites: cellular and molecular mechanisms," Nature Reviews Immunology, vol. 3, no. 9, pp. 733-744, 2003. 

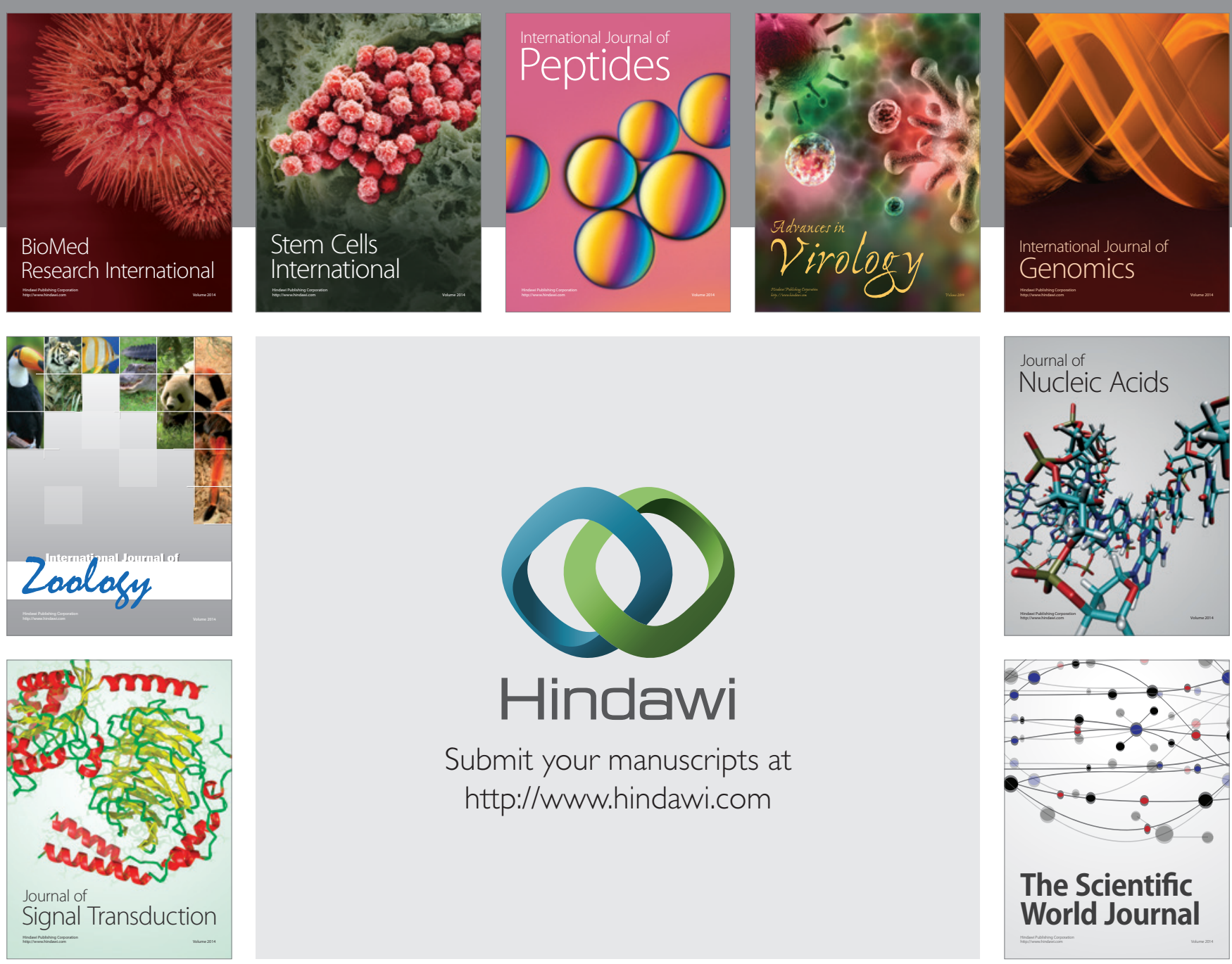

Submit your manuscripts at

http://www.hindawi.com
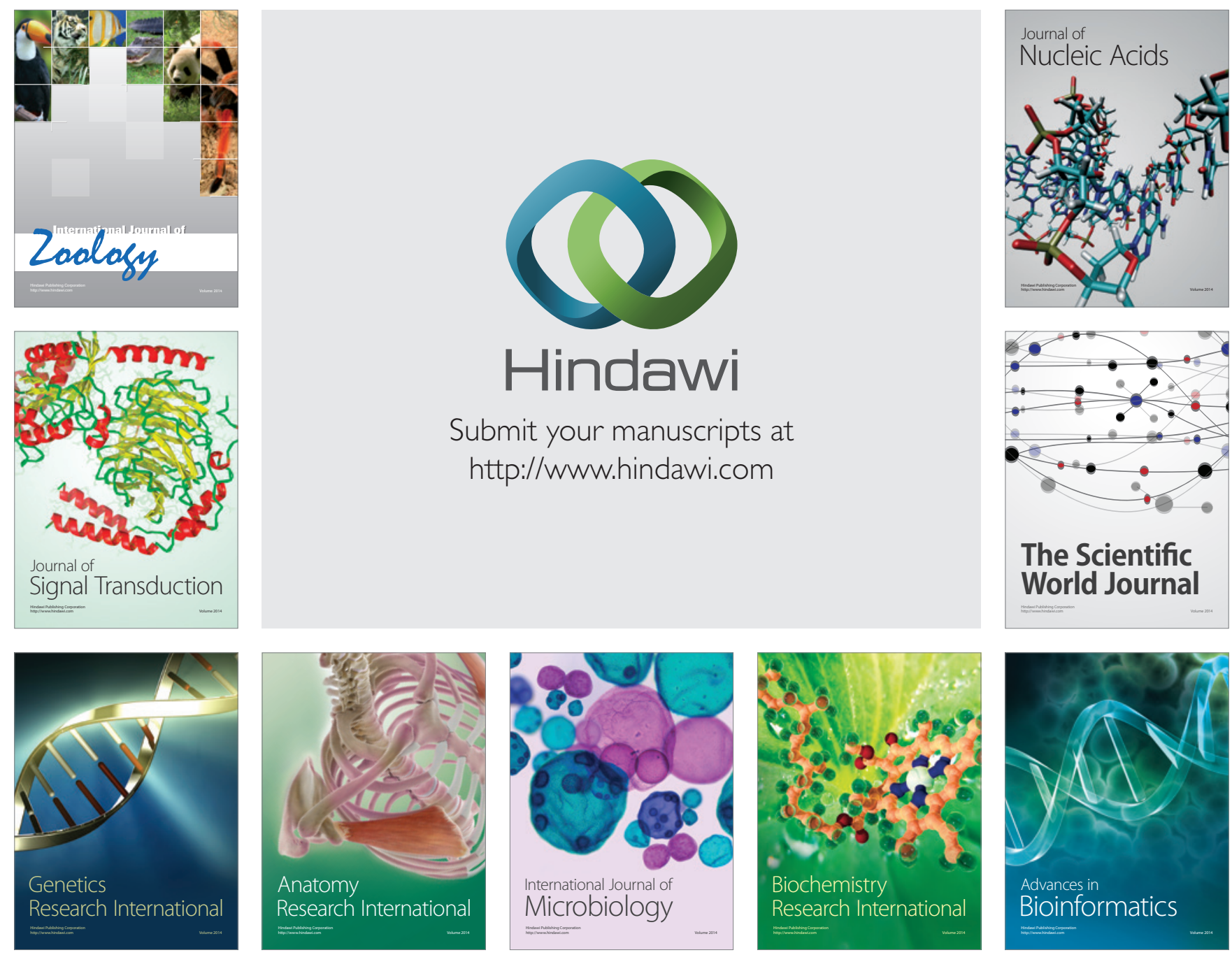

The Scientific World Journal
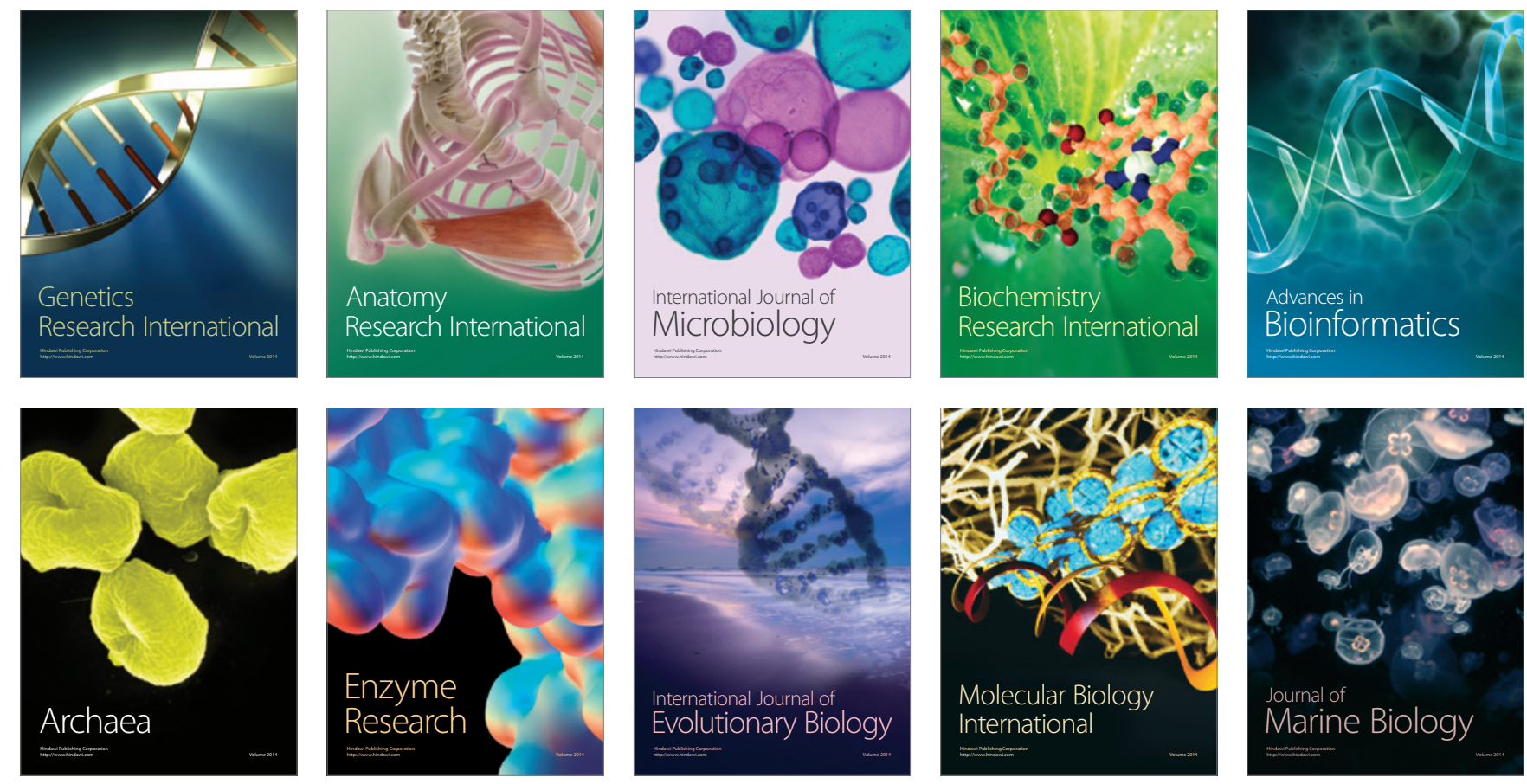